\title{
Immune-related disorders in families of children with inflammatory bowel disease - A prospective cohort study
}

\author{
Alyzée M Sibtain ${ }^{1}$, Donald Spady ${ }^{1}$ and Wael El-Matary ${ }^{2^{*}}$
}

\begin{abstract}
Background: The aim of this paper was to examine the prevalence of immune-related disorders in families of children with inflammatory bowel disease (IBD) compared to those without IBD.

Methods: Children $\leq 18$ years of age presenting to the IBD clinic between September 2007 and August 2009 with an established diagnosis of IBD were recruited. Age and sex matched controls without IBD were recruited. The study was a single-centre prospective cohort study. Outcome measures were prevalence of immune-based/ inflammatory diseases in families of both patients and controls.
\end{abstract}

Results: One hundred and eight children in each group were recruited. Asthma was the most frequently reported disease in families of the IBD patients (52.8\%) and controls (46.3\%). The prevalence of IBD in families of IBD patients was significantly higher than in those without IBD (OR 2.03, 95\% Cl 1.04-3.95).

Conclusions: The prevalence of immune-based disorders, as a group, in families of children with IBD was not significantly higher when compared to children without IBD.

\section{Background}

Inflammatory bowel diseases (IBD), Crohn's disease (CD) and ulcerative colitis (UC) are considered to be gastrointestinal luminal diseases of immune-dysregulation $[1,2]$. It is postulated that, IBD results from dysfunctional luminal epithelial barrier, and facilitated by an inappropriate innate and acquired immune response to commensal microorganisms that occur in individuals with appropriate genetic predisposition and susceptibility $[3,4]$. A genome study by Becker et al. demonstrated the presence of non-random overlapping of susceptibility loci among autoimmune diseases such as multiple sclerosis (MS), CD, psoriasis, asthma and type I diabetes (IDDM) [5]. Furthermore, there is considerable evidence that other immune-related disorders may be associated with bronchial asthma and rheumatoid arthritis [6-9].

The aim of this single-centre, hospital-based prevalence study was to examine the prevalence of family history of immune-based diseases among pediatric patients

\footnotetext{
* Correspondence: welmatary@yahoo.com

${ }^{2}$ Section of Pediatric Gastroenterology, Department of Pediatrics, University of Manitoba, Winnipeg, Canada

Full list of author information is available at the end of the article
}

with IBD compared to those without IBD. We hypothesized that there is an increased prevalence of inflammatory or immune-based disease among children diagnosed with IBD compared to children without IBD.

\section{Methods}

In a single centre cohort prospective study, children $\leq 18$ years of age presenting to the IBD clinic or emergency room, between September 2007 and August 2009, with an established diagnosis of IBD based on clinical, radiological and endoscopic evidence [10] were recruited (n $=108$ ). During the same time, children $\leq 18$ years presenting to the general gastrointestinal clinics with functional problems, gastroesophageal reflux or feeding problems, were recruited as controls $(n=108)$. IBD was excluded in the control group based on clinical, radiological, and endoscopy if indicated. Patients and controls were age and sex-matched. Family history of some immune-based and inflammatory disorders, including IBD, celiac, autoimmune thyroid disease (including Grave's and Hashimoto's), rheumatoid arthritis, bronchial asthma, autoimmune liver disease, multiple 
sclerosis (MS), and psoriasis or vitiligo from both patients and controls was collected during clinic visits.

A standardized questionnaire was used for each immune-based disease. Responses were provided from adults accompanying patients, most often patient's parents and recorded by the investigators during the interview. First-degree relatives were defined as parents and siblings of patients, and second-degree relatives were defined as uncles, aunts, and grandparents. For uncles and aunts it was clarified that the affected relative was a genetic relative, rather than related by marriage. All other relatives such as cousins and great grandparents were classified as distant relatives.

Measures were taken to reduce recall/interviews bias, included verification of the history taken during clinic visits against hospital records and taking family history every time patients or controls presented to IBD/GI clinics.

Informed consents were obtained from all participants after circulating study information sheets.

\section{Ethics}

The study protocol was approved by the Health Research Ethics Board of the University of Alberta, Edmonton, Canada.

\section{Statistical analysis}

Data were analyzed using Stata10 (TM) (Data Analysis and Statistical Software, Stata Corp, Austin, TX, USA). Summary statistics were obtained for all variables. Cross-tabulation analysis was made for all variables against disease (case/control status) to explore any possible relationships. An initial conditional logistic regression model was performed, with disease status (case or control) as the dependent variable and potential immune disorders as independent variables. Subsequently, only those variables that were significant at $\mathrm{p}<$ 0.05 level in the initial regression model were included in the final model.

\section{Results}

Asthma was the most frequently reported disease in both IBD patients (52.8\%) and the control group (46.3\%), as well as when CD (52.5\%) and UC (53.1\%) patients were examined separately [Table 1 ]. The second most frequently reported immune-mediated disease in IBD group was IBD (33.3\%), followed by autoimmune thyroid disease and rheumatoid arthritis (31\%). When examined separately (CD and UC), the second most frequently reported immune-mediated disorder in patients with $\mathrm{CD}$ after bronchial asthma was autoimmune thyroid disease (30.5\%), followed by rheumatoid arthritis (28.8\%) and IBD (27.1\%). In UC patients, the second most frequently reported immune-mediated disorder was IBD (40.8\%), followed by rheumatoid arthritis (34.7\%) and thyroid disease (32.7\%). In the control group, the second most commonly reported immunemediated disease was rheumatoid arthritis (33.3\%), followed by IBD and thyroid disease (24.1\%).

There was no statistically significant difference observed in the prevalence of immune-mediated diseases as a group in the family history of patients when compared to children without IBD [Table 2]. However, when the prevalence of IBD, celiac, thyroid disease, parathyroid disease, rheumatoid arthritis, bronchial asthma, autoimmune liver disease, MS, and psoriasis or vitiligo was examined individually, there was a statistically significant difference between IBD patients and controls, specifically in regards to IBD. The prevalence of IBD in the self-reported family history of IBD patients

Table 1 The Prevalence of immune-related disease in families of patients with IBD vs. controls

\begin{tabular}{|c|c|c|c|c|c|c|c|c|}
\hline \multirow{3}{*}{ Immune-Mediated Disorder } & \multicolumn{8}{|c|}{ Diagnosis } \\
\hline & \multicolumn{2}{|c|}{ IBD $(n=108)$} & \multicolumn{2}{|c|}{$C D(n=59)$} & \multicolumn{2}{|c|}{$U C(n=49)$} & \multicolumn{2}{|c|}{ Control $(n=108)$} \\
\hline & $\mathbf{N}$ & $\%$ & $\mathbf{N}$ & $\%$ & $\mathbf{N}$ & $\%$ & $\mathbf{N}$ & $\%$ \\
\hline Total Immune-Mediated & 89 & 82.4 & 47 & 79.7 & 42 & 85.7 & 92 & 85.2 \\
\hline IBD & 36 & 33.3 & 16 & 27.1 & 20 & 40.8 & 23 & 21.3 \\
\hline Celiac & 9 & 8.33 & 5 & 8.47 & 4 & 8.16 & 8 & 7.14 \\
\hline IDDM & 30 & 27.8 & 16 & 27.1 & 14 & 28.6 & 26 & 24.1 \\
\hline Thyroid & 34 & 31.5 & 18 & 30.5 & 16 & 32.7 & 45 & 24.1 \\
\hline Parathyroid & 2 & 1.85 & 1 & 1.69 & 1 & 2.04 & 1 & 0.93 \\
\hline Skin & 19 & 17.6 & 12 & 20.3 & 7 & 14.3 & 23 & 21.3 \\
\hline Joint & 34 & 31.5 & 17 & 28.8 & 17 & 34.7 & 36 & 33.3 \\
\hline Asthma & 57 & 52.8 & 31 & 52.5 & 26 & 53.1 & 50 & 46.3 \\
\hline MS & 8 & 7.41 & 3 & 5.08 & 5 & 10.2 & 9 & 8.33 \\
\hline Liver & 2 & 1.85 & 1 & 1.69 & 1 & 2.04 & 4 & 3.7 \\
\hline
\end{tabular}

Abbreviations:

IBD inflammatory bowel disease, CD Crohn's disease, UC ulcerative colitis, MS multiple sclerosis, IDDM insulin-dependent diabetes mellitus 
Table 2 Calculated statistical difference in the family history of immune-related disease in children with IBD

\begin{tabular}{|c|c|c|c|c|c|c|c|c|c|c|c|c|}
\hline \multirow{3}{*}{$\begin{array}{l}\text { Immune-Mediated Disease } \\
\text { Total Immune-Mediated Disease }\end{array}$} & \multicolumn{4}{|c|}{ IBD } & \multicolumn{4}{|c|}{$\mathrm{CD}$} & \multicolumn{4}{|c|}{ UC } \\
\hline & \multirow{2}{*}{$\begin{array}{c}\text { Odds Ratio } \\
0.82\end{array}$} & \multicolumn{2}{|c|}{$95 \% \mathrm{Cl}$} & \multirow{2}{*}{$\frac{\text { p-value }}{0.71}$} & \multirow{2}{*}{$\begin{array}{c}\text { Odds Ratio } \\
0.90\end{array}$} & \multicolumn{2}{|c|}{$95 \% \mathrm{Cl}$} & \multirow{2}{*}{$\frac{\text { p-value }}{1}$} & \multirow{2}{*}{$\frac{\text { Odds Ratio }}{0.68}$} & \multicolumn{2}{|c|}{$95 \% \mathrm{Cl}$} & \multirow{2}{*}{$\frac{\text { p-valu }}{0.76}$} \\
\hline & & 0.40 & 1.67 & & & 0.37 & 2.20 & & & 0.21 & 2.21 & \\
\hline IBD & 2.03 & 1.04 & 3.95 & $\leq 0.05$ & 0.87 & 0.31 & 2.42 & 0.79 & 5.30 & 1.50 & 18.67 & $\leq 0.05$ \\
\hline Celiac & 0.91 & 0.28 & 2.94 & 0.87 & 3.27 & 0.28 & 38.42 & 0.35 & 1.49 & 0.20 & 11.21 & 0.7 \\
\hline IDDM & 1.37 & 0.69 & 2.72 & 0.37 & 1.63 & 0.60 & 4.47 & 0.34 & 1.95 & 0.58 & 6.60 & 0.28 \\
\hline Thyroid & 0.57 & 0.29 & 1.07 & 0.08 & 0.71 & 0.30 & 1.70 & 0.44 & 0.29 & 0.08 & 1.023 & 0.05 \\
\hline Parathyroid & 1.56 & 0.10 & 24.36 & 0.75 & 0.15 & 0.002 & 9.61 & 0.38 & 0 & 0 & 3.86 & 0.99 \\
\hline Skin & 0.76 & 0.35 & 1.61 & 0.48 & 0.81 & 0.31 & 2.26 & 0.71 & 0.53 & 0.13 & 2.10 & 0.37 \\
\hline Joint & 0.88 & 0.46 & 1.68 & 0.69 & 0.79 & 0.31 & 2.02 & 0.62 & 1.38 & 0.42 & 4.54 & 0.59 \\
\hline Asthma & 1.32 & 0.75 & 2.30 & 0.34 & 1.84 & 0.87 & 3.89 & 0.11 & 1.08 & 0.34 & 3.37 & 0.9 \\
\hline MS & 0.887 & 0.28 & 2.71 & 0.81 & 1.47 & 0.23 & 9.41 & 0.61 & 0.60 & 0.11 & 3.36 & 0.56 \\
\hline Liver & 0.70 & 0.09 & 5.35 & 0.73 & 2.64 & 0.08 & 86.32 & 0.59 & 0.33 & 0.01 & 7.98 & 0.49 \\
\hline
\end{tabular}

Abbreviations:

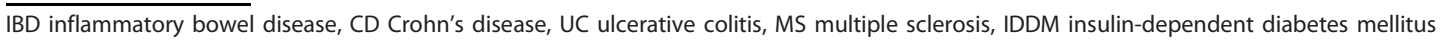

was higher than the prevalence of IBD in families of children without IBD (OR 2.03, 95\% CI 1.04-3.95) and the difference becomes higher in families with UC patients (OR 5.3, 95\% CI 1.5-18.67)

No statistically significant difference was observed in the prevalence of any of the other individually-examined immune-related diseases in families of children with IBD when compared to children without IBD.

The IBD patients were then categorized based on their diagnosis of CD or UC. There was no statistically significant difference in the prevalence of immune-mediated diseases as a group in families of children with UC or $\mathrm{CD}$ when compared to children without IBD.

\section{Discussion}

Over 30\% of older school children in Saskatchewan had wheezing in 12 months prior to a survey that was published in 1999 [11]. This prevalence of asthma in Canada is rising [12]. Asthma was the most frequently reported disease in families of children with IBD, similar to those without IBD. Interestingly, some reports indicated a higher prevalence of irritable bowel syndrome (a functional disorder) among young asthmatics [13].

Rheumatoid arthritis was the second most common disorder followed by IBD. This finding is consistent with results from previous studies examining co-morbid conditions among IBD patient $[7,14,15]$. In a cross-sectional study from Northern California, Weng et al identified asthma, rheumatoid arthritis and MS to be the most common co-morbidities among IBD patients, and suggested genetic linkage of immune-mediated diseases [16]. Thus, it is not unreasonable to suggest that these common co-morbidities as well as IBD may also be more common in families of pediatric patients with IBD.

The IBD occurrence in family of control group $24.1 \%$. The prevalence of IBD worldwide is approximately 396/
100,000 in a standard population [15]. This discrepancy between the prevalence in the control group and the standardized population may be due to the smaller sample size. Another explanation is the fact that the control group was recruited from patients with functional gastrointestinal problems rather than from general population.

The prevalence of self-reported immune-based disorders, as a group, in families of children with IBD was not significantly higher when compared to children without IBD. The relationship between autoimmune diseases and IBD has been examined, and several studies have shown an association between IBD and a positive family history specifically of MS, rheumatoid arthritis, and ankylosing spondylitis [16-18].

There was, on the other hand, a significantly higher prevalence of IBD in families of children with IBD, especially in children with UC compared to those without IBD. This finding is consistent with previous studies demonstrating a familial aspect of IBD $[9,15,19]$. A cohort study from six pediatric centres in the United States by Heyman et al reported a positive family history of IBD to be more common in UC patients [20]. However, there was not a significantly higher prevalence of IBD in families of children with CD. This is surprising, as $\mathrm{CD}$ is known to have a significant familial component as shown by genetic and population studies $[8,19]$. Nonetheless, a recent study by Hemminki et al. using The Multi-generation Register in Sweden demonstrated that, though present, concordant familial risks for both UC and CD were lower than previously cited [17].

The potential limitation of recall bias needs to be considered. Though patients were asked specifically about bronchial asthma, there are other types of wheezy chest syndromes that can be confusing. This may explain why asthma was not only the most commonly reported 
immune-mediated disease in both the patient and control group family histories, but also why there was no a significant difference in its prevalence between the two groups.

Measures taken to reduce recall/interviews bias, including verification of history taken against hospital records and taking family history very time patients or controls were presented to IBD/GI clinics.

Another limitation is that the control group was a group of children with functional gastrointestinal disorders like irritable bowel syndrome. It is better to recruit a control group with no gastrointestinal symptoms but there is no evidence to suggest that inflammatory bowel diseases are more prevalent in relatives of patients with functional gastrointestinal problems.

The presence of a significantly increased prevalence of positive family history of IBD in patients with IBD lends further support to the exploration of family history when determining the direction of investigations and diagnosis in children presenting with symptoms of IBD, as well as future screening and follow up is warranted.

\section{Conclusions}

The present study supports the available evidence documenting a significantly increased prevalence of positive family history of IBD, among pediatric IBD patients compared to children without IBD. However, the prevalence of self-reported immune-based disorders, as a group, in families of children with IBD was not significantly higher when compared to children without IBD.

\section{Abbreviations}

IBD:inflammatory bowel disease; CD: Crohn's disease; UC: ulcerative colitis; MS: multiple sclerosis; IDDM: insulin-dependent diabetes mellitus.

\section{Acknowledgements}

This work was supported by a grant from the Stollery Children's Hospital Foundation, Edmonton, Canada.

The authors thank Dr H Huynh, Dr J Turner, Dr R Persad and the Pediatric IBD team at the Stollery Children's Hospital for their help in recruiting patients.

\section{Author details}

${ }^{1}$ Department of Pediatrics, Stollery Children's Hospital, Faculty of Medicine, University of Alberta, Edmonton, Canada. ${ }^{2}$ Section of Pediatric Gastroenterology, Department of Pediatrics, University of Manitoba, Winnipeg, Canada.

\section{Authors' contributions}

AS interviewed patients and their families and wrote the manuscript. DS performed the statistical analysis. WEM conceived of the study, participated in its design and coordination, and helped to draft the manuscript. All authors read and approved the final manuscript

\section{Competing interests}

The author declares that they have no competing interests.
References

1. Cho $\mathrm{JH}$ : The genetics and immunopathogenesis of inflammatory bowel disease. Nat Rev Immunol 2008, 8:1474-1733.

2. Brown SJ, Mayer L: The immune response in inflammatory bowel disease. Am J Gastroenterol 2007, 102:2058-2069.

3. Strober W, Fuss I, Mannon P: The fundamental basis of inflammatory bowel disease. J Clin Invest 2007, 117:514-521.

4. Xavier RJ, Podolsky DK: Unraveling the pathogenesis of inflammatory bowel disease. Nature 2007, 448:427-434.

5. Becker KG, Simon RM, Bailey-Wilson ET, Freidlin B, Biddison WE, McFarland HF: Clustering of non-major histocompatibility complex susceptibility candidate loci in human autoimmune disease. Proc Nat Acad Sci USA 1998, 65:9979-9984.

6. Jose FA, Garnett EA, Vittinghoff E, Ferry GD, Winter HS, Baldassano RN, Kirschner BS, Cohen SA, Gold BD, Abramson O, Heyman MB: Development of extraintestinal manifestations in pediatric patients with inflammatory bowel disease. Inflamm Bowel Dis 2009, 15:63-38.

7. D'Amato M, Bruce S, Bresso F, Zucchelli M, Ezer S, Pulkkinen V: Neuriopeptide S Receptor 1 gene polymorphism is associated with susceptibility to inflammatory bowel disease. Gastroenterology 2007, 133:808-817

8. Schreiber S, Rosenstiel P, Albrect M, Hampe J, Krawczak M: Genetics of Crohn's disease, and archetypal inflammatory barrier disease. Nat Rev Genet 2005, 6:376-388.

9. Peeters M, Cortot A, Vermeire S, Colombel JF: Familial and sporadic inflammatory bowel disease: different entities? Inflamm Bowel Dis 2000, 4:314-20.

10. Turner D, Griffiths AM: Esophageal, gastric, and duodenal manifestations of IBD and the role of upper endoscopy in IBD diagnosis. Curr Gastroenterol Rep 2007, 6:475-478.

11. Habbick BF, Pizzichini MM, Taylor B, Rennie D, Senthilselvan A, Sears MR: Prevalence of asthma, rhinitis, eczema among children in 2 Canadian cities: the international study of asthma and allergies in childhood. CMAJ 1999, 160:1824-8.

12. Garner $R$, Kohen D: Changes in the prevalence of asthma among Canadian children. Health Rep 2008, 19:45-50.

13. Ekici A, Guliter S, Ekici M, Kalpaklioglu F, Kara T, Keles H, Tunckol M, Akin A, Kocyigit P: Irritable bowel syndrome in young and elderly patients with stable asthma. Dig Liver Dis 2005, 37:773-8.

14. Danese S, Semeraro S, Papa A, Roberto I, Scaldaferri F, Fedeli G: Extraintestinal manifestations of inflammatory bowel disease. World J Gastroenterol 2005, 11:7227-7236.

15. Halme L, Paavola-Sakki P, Turunen U, Lappalainen M, Farkkila M, Kontula K Family and twin studies in inflammatory bowel disease. World J Gastroenterol 2006, 21:3668-3672.

16. Weng X, Liu L, Barcellos LF, Allison JE, Herrinton LJ: Clustering of inflammatory bowel disease with immune mediated diseases among members of a Northern California-Managed Care Organization. Am J Gastroenterol 2007, 102:1429-1435.

17. Hemminki K, Li X, Sundquist K, Sandquest J: Familial association of inflammatory bowel diseases with other autoimmune and related diseases. Am J Gastroenterol 2010, 105:139-147.

18. Broadley SA, Deans J, Sawcer SJ, Clayton D, Compston DA: Autoimmune disease in first-degree relatives of patients with multiple sclerosis. Brain 2000, 123:1102-1111.

19. Baumgart DC, Carding SR: Inflammatory bowel disease: cause and immunobiology. Lancet 2007, 369:1627-1640.

20. Heyman MB, Kirschner BS, Gold BD, Ferry G, Baldassano R, Cohen SA: Children with early-onset inflammatory bowel disease (IBD): Analysis of a pediatric IBD consortium registry. J Pediatr 2005, 146:35-40.

doi:10.1186/1824-7288-37-49

Cite this article as: Sibtain et al:: Immune-related disorders in families of children with inflammatory bowel disease - A prospective cohort study. Italian Journal of Pediatrics 2011 37:49. 